\title{
Validity of mini-fluid challenge for predicting fluid responsiveness following liver transplantation
}

\author{
Ahmed Mukhtar ${ }^{1,6^{*}}$ D, Maha Awad², Mohamed Elayashy ${ }^{1}$, Amr Hussein 1, Gihan Obayah', Akram El Adawy ${ }^{1}$ \\ Mai Ahmed ${ }^{1}$, Hisham Abul Dahab', Ahmed Hasanin', Amr Elfouly ${ }^{3}$, Mostafa Abdo ${ }^{4}$, Amr Abdelaal $^{4}$ and \\ Jean Louis Teboul ${ }^{5}$
}

\begin{abstract}
Background: Mini-fluid challenge is a well tested and effective tool to predict fluid responsiveness under various clinical conditions. However, mini-fluid challenge has never been tested in patients with end-stage liver disease. This study investigated whether infusion of $150 \mathrm{ml}$ albumin $5 \%$ can predict fluid responsiveness in cirrhotic patients following liver transplant.

Methods: Fifty patients receiving living donor liver transplant were included in the analysis. Mini-fluid challenge composed of $150 \mathrm{ml}$ of albumin 5\% administered over $1 \mathrm{~min}$ in three consecutive 50-ml fluid boluses. An additional $350 \mathrm{ml}$ was then infused at a constant rate over $15 \mathrm{~min}$ (for a total of $500 \mathrm{ml}$ ). Stroke volume (SV) was measured as the product of the subaortic velocity time integral (VTI) and left ventricular outflow tract (LVOT) area. Fluid responsiveness was defined as an increase in SV by $\geq 15 \%$ after the infusion.

Results: Fifty patients were enrolled in the study. Fourteen patients were classified with Child A, 15 patients with Child B, and 21 patients with Child C cirrhosis. Thirty four patients were fluid responders and 16 patients were fluid non-responders. After $150 \mathrm{ml}$ of albumin 5\%, the SV increased significantly in our cohort. The area under receiver operating curve (AUROC) was 0.7 (95\% confidence interval [CI] 0.5-0.8, $P=0.005$ ). In subgroup analysis, the SV increased significantly after mini fluid challenge in the Child $\mathrm{A}$ group $(P=0.017)$ but not Child $\mathrm{B}$ or $C$ groups $(P=0.3$ and 0.29 , respectively). The AUROC for mini-fluid challenge in the Child A group was 0.86 ( $95 \%$ confidence interval $[C I] 0.6-0.9, P=0.0004$ ), while mini-fluid challenge failed to discriminate between responders and nonresponders in Child B and $C$ groups.
\end{abstract}

Conclusion: A mini-fluid challenge of $150 \mathrm{ml}$ albumin 5\% can predict fluid responsiveness in liver transplant patients with fair sensitivity and specifiicty. Subgroup analyis revealed that minifluid challenge can predict fluid responsiveness in patients with Child A cirrhosis but not patients with Child B or C cirrhosis.

Trial registration: NCT03396159. (Prospective registered). Initial registration date was 10/01/2018.

Keywords: Minifluid challenge, Liver transplantation, Fluid responsiveness

\footnotetext{
* Correspondence: Ahmed.Mukhtar@kasralainy.edu.eg

${ }^{1}$ Department of Anesthesia, Surgical Intensive Care and Pain Management,

Faculty of Medicine, Cairo University, Cairo, Egypt

${ }^{6}$ Cairo, Egypt

Full list of author information is available at the end of the article
}

(c) The Author(s). 2019 Open Access This article is distributed under the terms of the Creative Commons Attribution 4.0 International License (http://creativecommons.org/licenses/by/4.0/), which permits unrestricted use, distribution, and reproduction in any medium, provided you give appropriate credit to the original author(s) and the source, provide a link to the Creative Commons license, and indicate if changes were made. The Creative Commons Public Domain Dedication waiver (http://creativecommons.org/publicdomain/zero/1.0/) applies to the data made available in this article, unless otherwise stated. 


\section{Background}

Hemodynamic derangements are frequently observed during and after liver transplantation, mainly due to major blood loss, decreased venous return, and (or) reperfusion injury-induced vasodilatation. Fluid therapy is the cornerstone of hemodynamic management for unstable patients. The main goals of fluid therapy are to increase cardiac output $(\mathrm{CO})$ and improve tissue perfusion.

The concept of fluid responsiveness has been suggested as a guide for fluid administration in critically ill patients to avoid over- and under-fluid resuscitation. Fluid responsiveness is defined as the ability of the left ventricle to increase stroke volume (SV) after fluid administration [1, 2]. Several methods have been suggested to detect fluid responsiveness. Of these methods, some that depend on heart-lung interactions have been shown to be effective during intraoperative management of liver transplantation patients. However, during the postoperative period, these methods are not feasible in patients with spontaneous breathing activity. In these cases, mini-fluid challenge has emerged as an alternative for preload challenge [3].

Several studies have investigated the value of mini-fluid challenge as a predictor of subsequent fluid infusion under different clinical conditions $[4,5]$. However, none of these studies included patients with end-stage liver disease ESLD. Patients with ESLD have abnormal blood volume distribution, with a substantial fraction of this volume in the splanchnic circulation [6]. Rapid volume loading in these patients has little impact on CO because a large proportion of infused fluid is shifted to the splanchnic system. As a result, aggressive volume expansion to maintain tissue perfusion in liver transplantation recipients may be associated with volume overload and increased risks of poor graft function and postoperative morbidity [7]. Moreover, not all patients with ESLD exhibit the same response to changes in blood volume. Healthy individuals and patients with Child A cirrhosis differ in their response to blood volume expansion compared to patients with Child B and C cirrhosis [8].

The primary objective of the present study was to investigate the validity of mini-fluid challenge as a predictor of fluid responsiveness in patients with ESLD after liver transplantation. The seconadry objective was to examine the consistency of this validity among different Child classification. Our hypothesis was that mini-fluid challenge would not be a valid tool to predict fluid responsiveness in cirrhotic patients after liver transplant.

\section{Methods}

This prospective observational study was designed to enroll 50 consecutive living donor liver transplant (LDLT) patients. The indications for LDLT in our center include; patients with decompensated liver cirrhosis, liver cirrhosis with MELD score more than 15, and patients with hepatocellular carcinoma within transplant criteria. The study protocol was approved by the Research Ethics Committee of Cairo University Hospital and informed consent was obtained from all patients. The study is registered at clinical trial.gov (NCT03396159) and conducted from January 2018 to May 2018. Patients < 18 years of age, poor echocardiographic window, patient with CVP $>12$ or lung congestion or those with acute fulminant liver failure were excluded from the study.

Before ICU admission, a $20 \mathrm{G}$ radial artery catheter was inserted to measure arterial pressure and a triple lumen central venous catheter was inserted into the right jugular vein to measure central venous pressure (CVP). We typically used a fast track protocol, and all patients were extubated at the end of the operation inside the operating room.

Upon admission to the intensive care unit (ICU), patients were maintained in a supine position and pressure transducers were referenced to the level of the tricuspid valve and zeroed to atmospheric pressure after calibration.

\section{Study protocol}

All measurements were performed in the supine position within $1 \mathrm{~h}$ after arrival in the ICU. Hemodynamic measurements were performed at baseline. The fluid challenge was administered intravenously via a specific venous line. Mini-fluid challenge consisted of $150 \mathrm{ml}$ albumin 5\% delivered over $1 \mathrm{~min}$ as three consecutive $50-\mathrm{ml}$ fluid boluses with no time delay between them. The remaining $350 \mathrm{ml}$ was infused at a constant rate using preset electrical infusion pump over $15 \mathrm{~min}$ (for a total infusion volume of 500 $\mathrm{ml})$. Three sets of SV measurements were obtained: The first set was obtained before the mini-fluid challenge, the second set $1 \mathrm{~min}$ after the mini-fluid challenge, and the third set immediately after the infusion of the remaining $350 \mathrm{ml}$ (total $=500 \mathrm{ml}$ ) given over $15 \mathrm{~min}$. Fluid responsiveness was defined as an increase in SV by $15 \%$ after the infusion of $500 \mathrm{ml}$ albumin $5 \%$, thus separating the studied population into responders and non-responders. This cut of value was chosen as described previously [5].

Echocardiographic assessment was performed by an accredited physician (level 3) using a GE Vivid E9 echocardiographer. The assessor was blinded to Child-Pugh class of the patients. Stroke volume was measured as the product of the subaortic velocity time integral (VTI) and left ventricular outflow tract (LVOT) diameter. The VTI was obtained by placing the pulse wave Doppler sampling cursor in the middle of the LVOT immediately proximal to the aortic valve in the apical five-chamber view. For each step of the study, VTI was measured in triplicate and averaged for the determination of the VTI value. The LVOT diameter was measured for each patient as the distance between the inflection points at the 
base of the aortic valve cusps from the left parasternal long-axis view during systole. Finally, SV was calculated as VTI $\times$ LVOT area.

Primary endpoint in this study was the sensitivity and specificity of minifluid challenge to assess fluid responsiveness in cirrhotic patients.

\section{Statistical analysis and sample size calculation}

Sample size was calculated to detect an area under the receiver operating curve (AUROC) of 0.75 with null hypothesis $A U R O C=0.5$. Considering that the ratio of responders admitted to our unit is about half that of non-responders, a minimum of 42 patients including at least 14 responders are needed for a study power of $80 \%$ and an alpha error of 0.05 .

Data are expressed as mean (with standard deviation) unless specified otherwise. Continuous variables were compared by one-way ANOVA and post hoc Tukey tests, while categorical variables were compared by $x^{2}$ test. Paired variables within each group (before and after mini-fluid challenge) were compared using the Wilcoxon matched-pairs test. To compare the performance of mini-fluid challenge for assessing fluid responsiveness among subgroups (Child cirrhosis classes), receiver operating characteristic (ROC) curves were constructed and the area under the curve (AUC) calculated. MedCalc version 12.1.4.0 (MedCalc Software bvba, Mariakerke, Belgium) generated values with the highest sensitivity and specificity (Youden index). All statistical analyses were performed using SPSS for Windows version 15.0 (SPSS Inc., Chicago, IL, USA).

\section{Results}

Out of 60 patients screened, 55 met the inclusion criteria and fifty patients were available for final analysis. The reason for exclusion in all cases was a poor echocardiographic window. Fourteen patients had Child A cirrhosis, 15 Child B, and 21 patients Child C cirrhosis.
Age, sex, and BMI were comparable among the three groups. However, Model for End-Stage Liver Disease (MELD) score was significantly lower in the Child A group compared to both Child B and C groups (Table 1). All patients had preoperative good cardiac function with estimated ejection fraction between 55 and $65 \%$. Two patients with child $\mathrm{C}$ cirrhosis had history of preoperative ICU admission and none of studied population required renal replacement therapy before liver transplantation. All patients were admitted to ICU after successful extubation inside the operating room. The intra-observer variability was $5 \%$. Thirty four patients were fluid responders and 16 patients were fluid non-responders. After $150 \mathrm{ml}$ of albumin $5 \%$, the SV increased significantly in our cohort. The AUROC was 0.7 (95\% confidence interval [CI] 0.5-0.8, $P=0.005)$. and the optimum cut-off value for predicting fluid responsiveness was $\geq 10 \%$. This cut-off value had $56 \%$ sensitivity and $87 \%$ specificity for distinghishing responders from non-responders. In subgroup analysis, the SV increased significantly in the Child A group $(P=0.017)$ but not Child $\mathrm{B}$ or $\mathrm{C}$ groups $(P=0.3$ and 0.29 , respectively) after mini fluid challenge. All other hemodynamic variables remained unchanged during and following mini-fluid challenge and after completion of $500 \mathrm{cc}$ albumin 5\% (Tables 2 and 3). After fluid challenge, the SV increased by more than $15 \%$ in 10 patients of the Child A group (71.4\%), 11 patients in the Child B group (73.3\%), and 13 patients in the Child C group (62\%). The AUC for mini-fluid challenge in the Child A group was 0.86 (95\% CI 0.6-0.9, $P=0.0004$ ) and the optimum cut-off value for predicting fluid responsiveness was $\geq 10 \%$. This cut-off value had $70 \%$ sensitivity and $100 \%$ specificity for distinghishing responders from non-responders. In contrast to the Child A group, mini-fluid challenge failed to discriminate between responders and non-responders in the Child $\mathrm{B}$ and C groups (Table 4). The change in CVP after $150 \mathrm{cc}$ loading did not discriminate between responders and non-responders in any of the three groups.

Table 1 Patient characteristics

\begin{tabular}{|c|c|c|c|c|c|}
\hline & All patients $(n=50)$ & Child A $(n=14)$ & Child B $(n=15)$ & Child C $(n=21)$ & $P$-value \\
\hline Age (years) & $52(45-57)$ & $51(45-57)$ & $52(42-55)$ & $56(48-60)$ & 0.23 \\
\hline Sex M/F & $46 / 4$ & $14 / 0$ & $13 / 2$ & $19 / 2$ & 0.39 \\
\hline BMI & $26(24-29)$ & $27(23-29)$ & $26(23-26)$ & $26(24-29)$ & 0.35 \\
\hline MELD & $13(11-17)$ & $10(9-11)^{*}$ & $16(12-17)$ & $17(13-19)$ & $<0.001$ \\
\hline Graft weight (g) & $835(660-950)$ & $865(615-1022)$ & $690(620-800) \dagger$ & $870(740-990)$ & 0.012 \\
\hline Intraoperative use of vasopressor & $20(40 \%)$ & 0 & $2(13 \%)$ & $18(86 \%)^{*}$ & $<0.001$ \\
\hline Intraoperative use of colloid (ml) & $2000(1000)$ & $1500(500)$ & $1700(650)$ & $2000(900)$ & 0.14 \\
\hline ICU stay (days) & $7(7-10)$ & $7(7-10)$ & $7(7-14)$ & $7(7-10)$ & 0.9 \\
\hline Hospital stay (days) & $21(21-21)$ & $21(21-22)$ & $21(21-30)$ & $21(21-21)$ & 0.78 \\
\hline
\end{tabular}

Data are presented as median (IQR) or ratio

$B M I$ body mass index, MELD Model for end-stage liver disease, ICU intensive care unit

${ }^{a}$ denotes significant compared to the other two groups. ${ }^{\text {b }}$ denotes significant compared to CHILD C. $p<0.05$ 
Table 2 Hemodynamic variables at baseline and after mini-fluid challenge

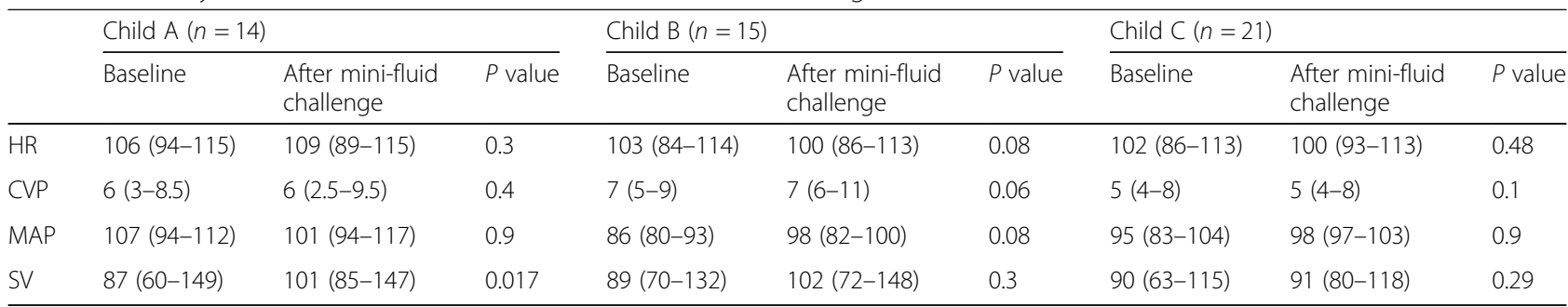

\section{Discussion}

The main finding of the present study is that a mini-fluid challenge ( $150 \mathrm{ml}$ of albumin $5 \%$ ) is sufficient to assess fluid responsiveness in patients with Child A cirrhosis but not Child B or C cirrhosis.

We found that a SV change $>10 \%$ during mini-fluid challenge following liver transplant asess fluid responsiveness in Child A cirrhosis patients with $70 \%$ sensitivity and $100 \%$ specificity. To the best of our knowledge, this is the first study to investigate mini-fluid challenge testing in patients with ESLD. Patients with ESLD not only have an abnormally high blood volume, but also an abnormal blood volume distribution. However, not all patients with ESLD exhibit the same response to changes in blood volume. Healthy individuals and patients with Child A cirrhosis differ in their response to blood volume expansion compared to patients with Child B and C cirrhosis. Volume expansion resulted in significantly increased central blood volume in patients with Child A cirrhosis compared to patients with Child $\mathrm{B}$ or $\mathrm{C}$ cirrhosis, [8] which may explain the difference in patient response to mini-fluid challenge observed in the present study. The plausible mechanism of this finding was that patients with Child A have less severe alteration in vasomotor and venous capicitance compared to Child $B$ and $C$ [9]. Thus, mini fluid bolus $(150 \mathrm{~mL})$ could increase the mean systemic filling pressure in patients with Child A but not in patients with Child B or C.

In the early stages of our liver transplant program, we typically extubated the patient $6-8 \mathrm{~h}$ after admission to the ICU [10]. However, we changed our protocol 8 years ago and more than $90 \%$ of patients are now extubated successfully inside the operating room. This makes the assessment of fluid responsiveness difficult because all dynamic indices, including pulse pressure variation and stroke volume variation, are unreliable during spontaneous breathing. Mini-fluid challenge and passive leg raising remain the only available tests for spontaneously breathing patients.

There is currently great interest in using fluid responsiveness as determined by mini-fluid challenge to guide transfusion, particularly to avoid volume overloading. While several studies have tested the concept of mini-fluid challenge, these investigations used different volumes and fluid types $[7,11,12]$. In the first such study, Muller et al. investigated the validity of mini-fluid challenge among 39 critically ill ventilated patients with acute circulatory failure and found that an increase in aortic blood flow $\geq 10 \%$ after rapid infusion of $100 \mathrm{ml}$ hydroxyethyl starch predicted fluid responsiveness. ${ }^{7}$ More recently, Aya et al. found that $4 \mathrm{ml} /$ $\mathrm{kg}$ was the minimum volume required to evoke a significant hemodynamic effect [10]. Smorenberg et al. reported that $150 \mathrm{ml}$ of hydroxyethyl starch reliably predicted fluid responsiveness after cardiac surgery [13]. In our study, we used the same volume $(150 \mathrm{ml})$ of albumin $5 \%$ and found this amount to be effective only in patients with Child A cirrhosis. Thus, a higher-volume mini-fluid challenge may be required for patients with advanced cirrhosis.

The concept of using predefined criteria for increase of $\mathrm{CO}$ after fluid challenge to differentiate between responder and non-responder depends on the starling mechanism. However, it is worth mentioning that $\mathrm{CO}$ changes during fluid bolus is affected not only by preload but also by afterload. Rapid fluid infusion decrease systemic vascular resistance by hemodilution. One study found that infusion of 11 of colloid caused $20 \%$ increase in blood volume, 20\% decrease in hemoglobin, and $40 \%$ increase in CO [14]. The increase in CO in their study is probably due to not only an increase in preload but also a decrease in afterload.

Table 3 Hemodynamic variables at baseline and after completion of $500 \mathrm{cc}$ fluid challenge

\begin{tabular}{|c|c|c|c|c|c|c|c|c|c|}
\hline & \multicolumn{3}{|c|}{ Child A $(n=14)$} & \multicolumn{3}{|c|}{ Child B $(n=15)$} & \multicolumn{3}{|c|}{ Child C $(n=21)$} \\
\hline & Baseline & After fluid challenge & $P$ value & Baseline & After fluid challenge & $P$ value & Baseline & After fluid challenge & $P$ value \\
\hline$\overline{\mathrm{HR}}$ & $106(94-115)$ & $104(89-111)$ & 0.3 & $103(84-114)$ & $103(86-110)$ & 0.09 & $102(86-113)$ & $98(88-110)$ & 0.45 \\
\hline CVP & $6(3-8.5)$ & $5(4-9.5)$ & 0.8 & $7(5-9)$ & $9(5-12)$ & 0.06 & $5(4-8)$ & $6(5-8)$ & 0.15 \\
\hline MAP & $107(94-112)$ & $101(95-119)$ & 0.9 & 86 (80-93) & $93(83-103)$ & 0.07 & $95(83-104)$ & $96(86-102)$ & 0.5 \\
\hline SV & $96(58-152)$ & $111(94-153)$ & 0.002 & $89(68-136)$ & $114(92-165)$ & 0.004 & $90(63-115)$ & $110(81-132)$ & 0.001 \\
\hline
\end{tabular}


Table 4 Comparison of the ability of mini-fluid challenge to assess fluid responsiveness among the three groups

\begin{tabular}{|c|c|c|c|c|c|c|}
\hline & $\begin{array}{l}\text { AUC of ROC } \\
\text { curve }\end{array}$ & $95 \% \mathrm{Cl}$ & $\begin{array}{l}\text { Cut-off } \\
\text { value }\end{array}$ & $\begin{array}{l}\text { Sensitivity } \\
(\%)\end{array}$ & $\begin{array}{l}\text { Specificity } \\
(\%)\end{array}$ & $P$-value \\
\hline Child A & $0.86^{*}$ & $0.6-0.9$ & $10 \%$ & 70 & 100 & 0.0004 \\
\hline Child B & 0.7 & $0.4-0.9$ & $16 \%$ & 100 & 50 & 0.26 \\
\hline Child C & 0.6 & $0.4-0.8$ & $7 \%$ & 58 & 87 & 0.2 \\
\hline
\end{tabular}

$P$ value denotes difference of AUC vs AUC of 0.5

$A U C$ area under the curce, $R O C$ receiver operating characteristic

*denotes statistical significance $(P<0.05)$

In our study, we defined fluid responsiveness as an increase in SV by $15 \%$ after infusion of $500 \mathrm{ml}$ albumin $5 \%$. Despite that the concept of fluid responsiveness has been emerged 20 years ago, there are still considerable variabilities in the definition, implementation, and assessment of fluid challenge. Recent systematic review found that $60 \%$ of the studies defined fluid responsiveness as an increase of $\geq 15 \%$ of CI or CO immediately after fluid challenge [15].

Albumin 5\% infusion was chosen to test fluid responsiveness instead of hydroxyethyl starch because we have previously shown that this solution is as effective for volume expansion [16]. Moreover, the safety of hydroxyethyl starch has been questioned and we have abandoned its use in the postoperative period [17].

Our study had several limitations. First, all our patients had excellent cardiac function during the early. Postoperative period, so we cannot extrapolate our findings to patients with poor cardiac function. Second, assessment of minifluid using echocardiography is challenging because the minifluid challenge induces small changes in cardiac output which may miss some true responders, however, we used single operator to limit the effect of interobserver variability.

\section{Conclusion}

A mini-fluid challenge of $150 \mathrm{ml}$ albumin $5 \%$ can predict fluid responsiveness in liver transplant patients with fair sensitivity and specifiicty. Subgroup analyis revealed that minifluid challenge can predict fluid responsiveness in patients with Child A cirrhosis but not in patients with Child B or C cirrhosis.

\section{Abbreviations}

AUROC: Area under receiver operating curve; CO: Cardiac output; CVP: Central venous pressure; ESLD: End stage liver disease; ICU: Intensive care unit; LVOT: Left ventricular outflow tract; MELD: Model for end stage liver disease; SV: Stroke volume; VTI: Velocity time integral

\section{Acknowledgments}

Not applicable.

\section{Funding}

This study has been funded by Cairo University Hospitals. The funding body contributed with research equipment. They did not have any influence on study design, data collection, analysis and interpretation of study data, or writing of the manuscript.

\section{Availability of data and materials}

Data are available from the authors upon reasonable request after permission of Alexandria University.

\section{Authors' contributions}

AM made substantial contribution to the study design and data analysis. MAwad, ME, AHussein, GO, were responsible for data collection, manuscript drafting. AEA, MAhmed, HA, AHasanin, AE, MAbdo, were responsible for data interpretation and analysis. AA and JT revised the manuscript critically for important intellectual content. All authors have read and approved the final manuscript.

\section{Ethics approval and consent to participate}

The study was approved by Research Ethic Committee (REC) at Cairo University (N-6-2018). All patients gave their written informed consent prior to study inclusion.

Study registered in clinicaltrial.gov NCT03396159. and initial registration date was 10/01/2018.

Consent for publication

Not applicable.

\section{Competing interests}

The authors declare that they have no competing interests.

\section{Publisher's Note}

Springer Nature remains neutral with regard to jurisdictional claims in published maps and institutional affiliations.

\section{Author details}

${ }^{1}$ Department of Anesthesia, Surgical Intensive Care and Pain Management, Faculty of Medicine, Cairo University, Cairo, Egypt. ${ }^{2}$ Department of Critical Care, Faculty of Medicine, Cairo University, Cairo, Egypt. ${ }^{3}$ Department of Internal Medicine, Faculty of Medicine, Helwan University, Cairo, Egypt. ${ }^{4}$ Department of Surgery, Ainshams University, Cairo, Egypt. ${ }^{5}$ Medical ICU, Bicêtre Hospital, Paris-South University, Paris, France. ${ }^{6}$ Cairo, Egypt.

Received: 5 February 2019 Accepted: 3 April 2019

Published online: 13 April 2019

\section{References}

1. Hasanin A. Fluid responsiveness in acute circulatory failure. J Intensive Care. 2015:3:50.

2. Huang C, Fu J, Hu H, Kao K, Chen N, Hsieh M, et al. Prediction of fluid responsiveness in acute respiratory distress syndrome patients ventilated with low tidal volume and high positive end-expiratory pressure* ${ }^{*}$ Crit Care Med. 2008;36:2810-6.

3. Monnet X, Rienzo M, Osman D, Anguel N, Richard C, Pinsky MR, et al. Passive leg raising predicts fluid responsiveness in the critically ill. Crit Care Med. 2006;34:1402-7.

4. Guinot PG, Bernard E, Defrancq F, Petiot S, Majoub Y, Dupont $H$, et al. Minifluid challenge predicts fluid responsiveness during spontaneous breathing under spinal anaesthesia: an observational study. Eur J Anaesthesiol. 2015; 32:645-9.

5. Muller L, Toumi M, Bousquet P-J, Riu-Poulenc B, Louart G, Candela D, et al. An increase in aortic blood flow after an infusion of $100 \mathrm{ml}$ colloid over 1 mnute can predict fluid responsiveness. Anesthesiology. 2011;115:541-7.

6. Kiszka-Kanowitz M, Henriksen JH, Moller S, Bendtsen F. Blood volume distribution in patients with cirrhosis: aspects of the dual-head gammacamera technique. J Hepatol. 2001:35:605-12.

7. Bennett-Guerrero E, Feierman DE, Barclay GR, Parides MK, Sheiner PA, Mythen MG, et al. Preoperative and intraoperative predictors of postoperative morbidity, poor graft function, and early rejection in 190 patients undergoing liver transplantation. Arch Surg. 2001;136:1177-83.

8. Moller S, Bendtsen F, Henriksen $\mathrm{JH}$. Effect of volume expansion on systemic hemodynamics and central and arterial blood volume in cirrhosis. Gastroenterology. 1995;109:1917-25. 
9. Hadengue A, Moreau R, Gaudin C, Bacq Y, Champigneulle B, Lebrec D. Total effective vascular compliance in patients with cirrhosis: a study of the response to acute blood volume expansion. Hepatology. 1992;15:809-15.

10. Abofetouh F, Khater Y, Mukhtar A, Salah M, Khedr H, Hamed H, et al. Perioperative management in adult and pediatric living related liver transplantation: an Egyptian experience. Int Anesthesiol Clin. 2006;44:127-36.

11. Aya HD, Rhodes A, Chis Ster I, Fletcher N, Grounds RM, Cecconi M. Hemodynamic effect of different doses of fluids for a fluid challenge: a quasi-randomized controlled study. Crit Care Med. 2017;45:161-8.

12. Biais $M$, De Courson $H$, Lanchon R, Pereira B, Bardonneau G, Griton M, et al. Mini-fluid challenge of $100 \mathrm{ml}$ of crystalloid predicts fluid responsiveness in the operating room. Anesthesiology. 2017;127:450-6.

13. Smorenberg A, Cherpanath TGV, Geerts BF, de Wilde RBP, Jansen JRC, Maas $\mathrm{J}$, et al. A mini-fluid challenge of $150 \mathrm{~mL}$ predicts fluid responsiveness using ModelflowRpulse contour cardiac output directly after cardiac surgery. J Clin Anesth. 2018;46:17-22.

14. Ueyama H, He YL, Tanigami H, Mashimo T, Yoshiya I. Effects of crystalloid and colloid preload on blood volume in the parturient undergoing spinal anesthesia for elective cesarean section. Anesthesiology. 1999;91:1571-157 6

15. Messina A, Longhini F, Coppo C, Pagni A, Lungu R, Ronco C, et al. Use of the fluid challenge in critically ill adult patients: a systematic review. Anesth Analg. 2017:5:1532-43.

16. Mukhtar A, Aboulfetouh F, Obayah G, Salah M, Emam M, et al. The safety of modern hydroxyethyl starch in living donor liver transplantation: a comparison with human albumin. Anesth Analg. 2009;109:924-30.

17. Myburgh JA, Finfer S, Bellomo R, Billot L, Cass A, Gattas D, et al.

Hydroxyethyl starch or saline for fluid resuscitation in intensive care. N Engl J Med. 2012;367:1901-11.

Ready to submit your research? Choose BMC and benefit from:

- fast, convenient online submission

- thorough peer review by experienced researchers in your field

- rapid publication on acceptance

- support for research data, including large and complex data types

- gold Open Access which fosters wider collaboration and increased citations

- maximum visibility for your research: over $100 \mathrm{M}$ website views per year

At $\mathrm{BMC}$, research is always in progress.

Learn more biomedcentral.com/submissions 